\title{
Choosing Wisely in the UK: the Academy of Medical Royal Colleges' initiative to reduce the harms of too much medicine
}

\author{
(c) (1) (8) OPEN ACCESS
}

A Malhotra and colleagues explain how and why a US initiative to get doctors to stop using interventions with no benefit is being brought to the UK

\author{
A Malhotra consultant clinical associate ${ }^{1}$, D Maughan Royal College of Psychiatrists sustainability \\ fellow $^{2}$, J Ansell advanced trainee in general surgery ${ }^{3}$, R Lehman senior research fellow ${ }^{4}, \mathrm{~A}$ \\ Henderson chief executive ${ }^{1}$, M Gray director ${ }^{5}$, T Stephenson former chair ${ }^{16}$, S Bailey chair ${ }^{1}$ \\ ${ }^{1}$ Academy of Medical Royal Colleges, London, UK; ${ }^{2}$ Centre For Sustainable Healthcare, Oxford, UK; ${ }^{3}$ Welsh Institute for Minimal Access Therapy, \\ Cardiff Medicentre, Cardiff, UK; ${ }^{4}$ Department of Primary Health Care, University Of Oxford, Oxford, UK; ${ }^{5}$ Better Value Healthcare, Oxford, UK; \\ ${ }^{6}$ Institute of Child Health, London, UK
}

The idea that some medical procedures are unnecessary and can do more harm than good is as old as medicine itself. In Mesopotamia 38 centuries ago, Hammurabi proclaimed a law threatening overzealous surgeons with the loss of a hand or an eye. In 1915, at the height of a surgical vogue for prophylactic appendicectomy, Ernest Codman offended his Boston colleagues with a cartoon (figure $\Downarrow$ ) mocking their indifference to outcomes and asking, "I wonder if clinical truth is incompatible with medical science? Could my clinical professors make a living without humbug?" Looking at the rates of tonsillectomy in London boroughs in the 1930s, John Alison Glover discovered that they were entirely governed by the policy of school doctors and bore no relation to need or outcomes. ${ }^{1}$ John (Jack) Wennberg established the science of outcomes research when in 1973 he described patterns of gross variation in the use of medical and surgical procedures in the United States, which lacked any clinical rationale but was closely related to supply. ${ }^{2}$

Diagnosis drives treatment, and in recent years the term overdiagnosis has been used to describe various situations where diagnoses lead to unnecessary treatment, wasting resources while increasing patient anxiety. Overdiagnosis can be said to occur when "individuals are diagnosed with conditions that will never cause symptoms or death" often as a "consequence of the enthusiasm of early diagnosis."3 Overtreatment includes treatment of these overdiagnosed conditions. It also encompasses treatment that has minimal evidence of benefit or is excessive (in complexity, duration, or cost) relative to alternative accepted standards. ${ }^{45}$ A recent report by the Academy of Medical Royal Colleges argued that doctors have an ethical responsibility to reduce this wasted use of clinical resource because, in a healthcare system with finite resources, one doctor's waste is another patient's delay. ${ }^{6}$

\section{Choosing Wisely in the NHS}

Even before the inception of the NHS, the British tradition has generally been one of late adoption and cautious use of new medicines, procedures, and technologies. Nevertheless, the UK shows similar patterns of variation in use of medical and surgical interventions to those in the US, though less extreme in absolute terms. ${ }^{7}$ The National Institute for Health and Care Excellence (NICE) was set up in 1999 in part to address these unwarranted variations in clinical practice and has identified over 800 clinical interventions for potential disinvestment. ${ }^{8}$ However, engaging clinicians with stopping familiar or ingrained practices requires a different approach to that for introducing new treatments.

An initiative recently developed in the US and Canada called Choosing Wisely (www.choosingwisely.org) aims to change doctors' practice to align with best practice by getting them to stop using various interventions that are not supported by evidence, free from harm, and truly necessary, including those that duplicate tests or procedures already received. Choosing Wisely asks medical organisations (such as medical royal colleges in the UK) to identify tests or procedures commonly used in their specialty, the necessity of which should be questioned and discussed. These are compiled into lists, and the "top five" interventions for each specialty should not be used routinely or at all. ${ }^{9}$ So far, more than 60 US specialist societies have joined in the Choosing Wisely initiative. It has also been adopted by other countries, including Australia, Germany, Italy, 
Japan, Netherlands, and Switzerland—a clear sign that wasteful medical practices are a problem for all health systems. ${ }^{10}$

The Academy of Medical Royal Colleges, which represents all medical royal colleges in the UK, is launching a Choosing Wisely programme in collaboration with other clinical, patient, and healthcare organisations. Participating organisations will work together to develop top five lists of tests or interventions with questionable value. The academy, royal colleges, and partners, including The BMJ, will then promote dissemination of this information and Choosing Wisely conversations between clinicians and patients. These new conversations will rebalance discussions about the risks and benefits of tests and interventions, such that doctors and patients will be supported to acknowledge that a minor potential benefit may not outweigh potential harm, the minimal evidence base, and substantial financial expense and therefore that, sometimes, doing nothing might be the favourable option.

\section{Tackling the underlying causes of overtreatment}

A culture of "more is better," where the onus is on doctors to "do something" at each consultation has bred unbalanced decision making. This has resulted in patients sometimes being offered treatments that have only minor benefit and minimal evidence despite the potential for substantial harm and expense. This culture threatens the sustainability of high quality healthcare and stems from defensive medicine, patient pressures, biased reporting in medical journals, commercial conflicts of interest, and a lack of understanding of health statistics and risk. $^{11}$

The system has no incentive to restrict doctors' activity; the NHS in England has a system of payment by results, which in reality is often a payment by activity and encourages providers to do more both in primary and secondary care. General practice is increasingly pressured to focus less on open dialogue with patients about treatment options and more on fulfilling the demands of the Quality and Outcomes Framework (QOF, a pay for performance instrument) and adhering to local commissioning decisions.

The quality measures in both primary and secondary care are based on guidelines produced by NICE, but doctors should not consider these as tramlines because decisions need to be made with reference to individual patient circumstances, the wishes of the patient, clinical expertise, and available resources. Some people would choose to take a hypothetical pill with no side effects daily, even for a few weeks' gain in life expectancy, whereas others would prefer not to, even if they were told it would add 10 years to their lifespan. ${ }^{12}$ It is instructive to note that a large and comprehensive longitudinal study recently concluded that higher reported achievement incentivised under QOF has not reduced premature death in the population. ${ }^{13}$

We suggest that guideline committees should increasingly turn their efforts towards the production of tools that help clinicians to understand and share decisions on the basis of best evidence. Rather than prespecifying the outcome of such dialogue, and trying to get medicine "just right," they should try to ensure that decisions are based on the best match between what is known about the benefits and harms of each intervention and the goals and preferences of each patient. ${ }^{14}$

More informed decision making can also alleviate, perhaps disproportionate, fears for those patients who may not want treatment. ${ }^{15} \mathrm{~A}$ recent study revealed that when patients were told the lack of prognostic benefit for angioplasty, only $46 \%$ elected to go ahead with the procedure versus $69 \%$ who were not explicitly given this information. ${ }^{16}$ Responding to similar concerns about getting patients' consent for elective coronary angioplasty in the UK, NHS England's cardiology lead, Huon Gray, stated, "It is important that doctors are clear with their patients about this."

It is easy to misunderstand health statistics, and doctors can find themselves needing to manage unrealistic expectations of patients who may find it difficult to obtain reliable information. Communicating relative risks as opposed to absolute risk or numbers needed to treat can often unintentionally mislead. As Gerd Gigerenzer, director of Harding Centre for Risk Literacy in Berlin, summarised in 2009, "It is an ethical imperative that every doctor and patient understand the difference between absolute and relative risks, to protect patients against unnecessary anxiety and manipulation." 17

Doctors' health illiteracy is well documented. Misunderstanding of statistics often leads to a belief that screening is more beneficial than it actually is and, in some cases, to no acknowledgment of its potential harms. In a study of 150 gynaecologists, one third did not understand the meaning of a $25 \%$ risk reduction from mammography. Many believed that if all women were screened $25 \%$ of women (or 250 fewer out of every 1000) would die of breast cancer, when actually the best evidence based estimate is actually one less death per 2000 women (from Cochrane's analysis of randomised studies including 500000 women).

Both medical and surgical overtreatment can place patients at high risk of adverse events. ${ }^{18}$ Shared decision making can help to reduce this overtreatment ${ }^{19}$ and may be particularly beneficial to disadvantaged groups, significantly improving health outcomes and reducing health inequalities. ${ }^{20}$

\section{Potential limitations}

One of the major concerns about the development of top five lists in the US is the potential for individual societies to choose low hanging fruit. For example, the American Academy of Orthopaedic Surgeons included the use of an over the counter supplement but no major procedures, despite evidence of wide variation in elective knee replacement and arthroscopy rates among Medicare beneficiaries. ${ }^{21}$ Currently, there is also no evidence that lists reduce use of low value medical practices. ${ }^{22}$ One crucial and relevant marker of success would be universal awareness of the Choosing Wisely programme among doctors and patients. However, despite much publicity in the medical literature, a random telephone survey of 600 US doctors recently conducted by the American Board of Internal Medicine found that only $21 \%$ had heard of Choosing Wisely. ${ }^{23}$ The level of public awareness of the campaign, which is a fundamental component to its progress, has not been assessed.

Reducing wasteful and harmful healthcare will require commitment from both doctors and patients, in addition to objective evidence of effectiveness. The NHS already has good systems for evidence appraisal and health technology assessment, but better and simpler tools are needed to facilitate informed discussion in clinical settings. Without such robust and easily shared decision aids, systematically updated without bias, patients may be swayed by potential exaggerated claims in the media when new drugs or procedures are introduced. Lastly, shared decision making does not guarantee lower resource use $\mathrm{e}^{24}$; greater involvement of patients in deciding their care will require a new set of consultation skills as well as a better range of decision aids. 


\section{Call to action and next steps}

To ensure the development of a Choosing Wisely culture in clinical practice, the academy suggests:

- Doctors should provide patients with resources that increase their understanding about potential harms of interventions and help them accept that doing nothing can often be the best approach

- Patients should be encouraged to ask questions such as, "Do I really need this test or procedure? What are the risks? Are there simpler safer options? What happens if I do nothing?"

- Medical schools should ensure that students develop a good understanding of risk alongside critical evaluation of the literature and transparent communication. Students should be taught about overuse of tests and interventions. Organisations responsible for postgraduate and continuing medical education should ensure that practising doctors receive the same education

- Commissioners should consider a different payment incentive for doctors and hospitals

Support from the media and medical publications will be vital because the public education campaign is crucial to the programme's success. The academy will ensure that the programme is thoughtfully implemented and rigorously evaluated by demonstrating a reduction in wasteful practices within a fixed time scale. It will begin by asking specialty organisations to compile top five lists. All lists will be accompanied by an implementation plan and will be evaluated and monitored to assess their effect on reducing low value healthcare.

The academy has set up a steering group to provide policy advice and direction for the project. The group comprises individual experts, patient groups, college representatives and key stakeholders. It is time for action to translate the evidence into clinical practice and truly wind back the harms of too much medicine.

Competing interests: We have read and understood BMJ policy on declaration of interests and have no relevant interests to declare.

Provenance and peer review: Not commissioned; externally peer reviewed.
1 Glover A. The incidence of tonsillectomy in school children. Proc $R$ Soc Med 1938:31:1219-36. (Reprinted Int J Epidemiol 2008;37:09-19).

2 Wennberg J, Gittelsohn A, Small area variations in health care delivery: a population-based health information system can guide planning and regulatory decision-making. Science 1973;182:1102-8.

3 Welch HG, Schwartz L, Woloshin S. Overdiagnosed; making people sick in the pursuit of health. Beacon Press, 2012.

4 Hoffman A, Pearson SD. "Marginal medicine": targeting comparative effectiveness research to reduce waste. Health Aff (Millwood) 2009;28:w 710-8.

5 Djulbegovic B, Paul A. From efficacy to effectiveness in the face of uncertainty: indication creep and prevention creep. JAMA 2011;305:2005-6.

6 Academy of Royal Medical Colleges. Protecting resources, promoting value: a doctor's guide to cutting waste in clinical care. 2014. www.aomrc.org.uk/dmdocuments/Promoting\% 20value\%20FINAL.pdf.

7 NHS atlas of variation in healthcare. 2010. www.rightcare.nhs.uk/atlas/qipp_nhsAtlasLOW_261110c.pdf.

8 Garner S, Littlejohns P. Disinvestment from low value clinical interventions: NICEly done? BMJ 2011;343:d4519.

9 Hurley R. Can doctors reduce harmful medical overuse worldwide? BMJ 2014;349:g4289.

10 Levinson W1, Kallewaard M2, Bhatia RS, et al. "Choosing Wisely": a growing international campaign. BMJ Qual Saf 2015;24:164-7.

11 Gigerenzer G, Muir Gray JA. Better doctors, better patients, better decisions. Envisioning healthcare 2020. MIT Press, 2011.

12 Fontana $\mathrm{M}$, Asaria $\mathrm{P}$, Moraldo $\mathrm{M}$, et al. Patient-accessible tool for shared decision making in cardiovascular primary prevention: balancing longevity benefits against medication. Circulation 2014;129:2539-46. .

13 Kontopantelis E, Springate DA, Ashworth M, et al. Investigating the relationship between quality of primary care and premature mortality in England: a spatial whole-population study. BMJ 2015;350:h904.

14 Macdonald $\mathrm{H}$, Loder $\mathrm{E}$. Too much medicine: the challenge of finding common ground BMJ 2015;350:h1163.

15 Malhotra A. The whole truth about coronary stents: the elephant in the room. JAMA Intern Med 2014:174:1367-8.

16 Rothberg MB, Scherer L, Kashef M, et al. The effect of information presentation on beliefs about the benefits of elective percutaneous coronary intervention. JAMA Intern Med 2014;174:1623-9.

17 Gigerenzer G. Making sense of health statistics. Bull World Health Organ 2009;87:567.

18 Morgan DJ, Wright SM, Dhruva S. Update on medical overuse. JAMA Intern Med 2015;175:120-4.

19 Stacey D, Bennett CL, Barry MJ, et al. Decision aids for people facing health treatment or screening decisions. Cochrane Database Syst Rev 2011;10:CD001431.

20 Durand M-A, Carpenter L, Dolan H, et al. Do interventions designed to support shared decision-making reduce health inequalities? A systematic review and meta-analysis. PLOS ONE 2014;9: e94670.

21 Morden NE1, Colla CH, Sequist TD, Rosenthal MB. Choosing Wisely-the politics and economics of labeling low-value service. N Engl J Med 2014;370:589-92.

22 Clement $F$, Charlton B. Challenges in Choosing Wisely's international future: support, evidence, and burnout. JAMA Intern Med 2015. [Epub ahead of print.]

23 Perry Undem Research/Communication. Unnecessary tests and procedures in the health care system: what physicians say about the problem, the causes, and the solutions: results from a national survey of physicians. 2014. www.choosingwisely.org/wp-content/uploads/ 2015/04/Final-Choosing-Wisely-Survey-Report.pdf.

24 Walsh T, Barr PJ, Thompson R, et al. Undetermined impact of patient decision support interventions on healthcare costs and savings: systematic review. BMJ 2014;348:g188.

Accepted: 25 March 2015

\section{Cite this as: BMJ 2015:350:h2308}

This is an Open Access article distributed in accordance with the Creative Commons Attribution Non Commercial (CC BY-NC 4.0) license, which permits others to distribute, remix, adapt, build upon this work non-commercially, and license their derivative works on different terms, provided the original work is properly cited and the use is non-commercial. See: http://creativecommons.org/licenses/by-nc/4.0/. 


\section{Figure}

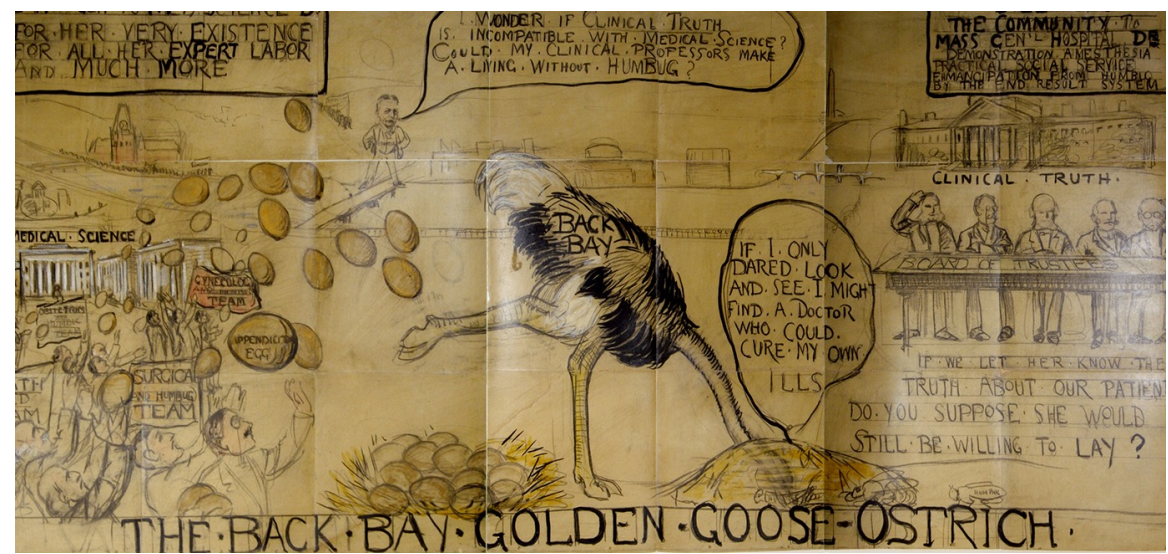

"Back Bay golden goose ostrich" cartoon

[Image: BOSTON MEDICAL LIBRARY] 